\title{
Transcriptome-Wide Identification, Evolutionary Analysis, and GA Stress Response of the GRAS Gene Family in Panax ginseng C. A. Meyer
}

\author{
Nan Wang ${ }^{1,2,+}$, Kangyu Wang ${ }^{1,2,+}$, Shaokun Li 1,2, Yang Jiang ${ }^{1,2}, \mathrm{Li} \mathrm{Li}^{1,2}$, Mingzhu Zhao ${ }^{1,2}$, \\ Yue Jiang ${ }^{1,2}$, Lei Zhu ${ }^{1,2}$, Yanfang Wang ${ }^{2,3}$, Yingjie Su ${ }^{1}$, Yi Wang ${ }^{1,2, *}$ and Meiping Zhang ${ }^{1,2, *}$ \\ 1 College of Life Science, Jilin Agricultural University, Changchun 130118, Jilin, China; \\ wangnanwg@sina.com (N.W.); kangyu.wang@jlau.ed.cn (K.W.); lishaokun1168@163.com (S.L.); \\ wky427@sina.com (Y.J.); lili910607@163.com (L.L.); zhaomingzhu0125@163.com (M.Z.); \\ jiangyue285431@163.com (Y.J.); zhulei0916@163.com (L.Z.); suyj0923@163.com (Y.S.) \\ 2 Research Center Ginseng Genetic Resources Development and Utilization, Changchun 130118, Jilin, China; \\ yfwang2014@163.com \\ 3 College of Chinese Medicinal Materials, Jilin Agricultural University, Changchun 130118, Jilin, China \\ * Correspondence: wanglaoshi0606@163.com (Y.W.); meiping.zhang@jlau.edu.cn (M.Z.) \\ + These two authors contributed equally to this work.
}

Received: 10 December 2019; Accepted: 24 January 2020; Published: 4 February 2020

\begin{abstract}
GRAS transcription factors are a kind of plant-specific transcription factor that have been found in a variety of plants. According to previous studies, GRAS proteins are widely involved in the physiological processes of plant signal transduction, stress, growth and development. The Jilin ginseng (Panax ginseng C.A. Meyer) is a heterogeneous tetraploid perennial herb of the Araliaceae family, ginseng genus. Important information regarding the GRAS transcription factors has not been reported in ginseng. In this study, 59 Panax ginseng GRAS (PgGRAS) genes were obtained from the Jilin ginseng transcriptome data and divided into 13 sub-families according to the classification of Arabidopsis thaliana. Through systematic evolution, structural variation, function and gene expression analysis, we further reveal GRAS's potential function in plant growth processes and its stress response. The expression of PgGRAS genes responding to gibberellin acids (GAs) suggests that these genes could be activated after application concentration of GA. The qPCR analysis result shows that four PgGRAS genes belonging to the DELLA sub-family potentially have important roles in the GA stress response of ginseng hairy roots. This study provides not only a preliminary exploration of the potential functions of the GRAS genes in ginseng, but also valuable data for further exploration of the candidate $P g G R A S$ genes of GA signaling in Jilin ginseng, especially their roles in ginseng hairy root development and GA stress response.
\end{abstract}

Keywords: Panax ginseng; GRAS transcription factor; DELLA sub-family; Evolutionary analysis; Gibberellin acid (GA); Stress response

\section{Introduction}

Ginseng is one of the most valuable medicinal plants in the Araliaceae family. Ginseng ingredients include saponins, polysaccharides, volatile ingredients, proteins, vitamins, and other compounds. The main active ingredient is ginsenosides. Asian regions, particularly China, Korea and Japan, are the major growing areas of ginseng. The Chinese ginseng is mainly distributed in Jilin Province and is commonly named as Jilin Ginseng. Ginseng has anti-thrombosis and anti-aging effects, prevents tumor angiogenesis and has other functions [1,2]. In recent years, the study of ginseng has become a hotspot for research. 
Transcription factors (TFs) are important regulatory factors affecting growth and development, physiological processes and regulation of the network in higher plants. Additionally, TFs are necessary in the regulation and control of gene expression [3,4]. Under normal circumstances, transcription factors are combined with other proteins or DNA sequences to perform their special functions. In recent years, many transcription factor families have been discovered, such as the WRKY (WRKYGQK domain), MYB (V-myb avian myeloblastosis viral oncogene homolog), AP2/ERF (Ethylene responsive element binding factor), bZIP (Basic region/leucine zipper motif) and GRAS (GAI RGA and SCR) families [5-8]. GRAS TFs are named after the acronyms of three members that were initially identified, GAI (GIBBERELLIN-ACID INSENSITIVE), RGA (REPRESSOR of gai1-3) and SCR (SCARECROW) [9,10]. Typically, the GRAS protein is composed of 400-770 amino acid residues [11,12]. GRAS proteins possess a highly conserved sequence at the end of the carboxyl terminus which contains several ordered motifs LHRI, VHIID, LHRII, PFYRE and SAW [13,14]. Among these conserved sequences, the VHIID sequence, which is essential for the interaction between proteins, is located in the middle of the leucine-rich LHR I motif and LHR II motif [15]. PFYRE and SAW may be associated with the structural integrity of the GRAS family. There is a significant difference in the amino terminus motif in the GRAS, which may determine the functional specificity of the GRAS proteins. In accordance with the length and difference of amino terminus in Arabidopsis thaliana, the GRAS proteins were divided into 17 sub-families [14]. The 17 sub-families were named as LISCL (lilium longiflorum scarecrow-like), PAT (phytochrome A signal transduction), DELLA (the DELLA motif containing protein), HAM (hairy meristem), LS (lateral suppressor), SCL3 (scarecrow-like 3), SCR (scarecrow), SHR (short root), DLT (dwarf and low-tillering), SCL32 (scarecrow-Like 32), SCL4/7 (scarecrow-like 4 and 7), NAP1 (nodulation signaling pathway 1), NSP2 (nodulation signaling pathway 2), RAM1 (reduced arbuscular mycorrhization 1), RAD1 (required for arbuscule development 1), SCLA (scarecrow-like-A) and OG-MIG1 (mycorrhiza-induced GRAS), respectively $[9,16]$. LISCL is involved in the regulation of microspore occurrence in anther by binding with the meiosis-associated promoter [2]. According to previous studies, the PAT of GRAS proteins has been involved in the early stages of photosensitive phytochrom A signaling transduction [17]. According to the previous study, we found that the HAM family was involved in the development of apical meristem and leaf axils of meristem tissue [18]. The LS sub-family is mainly involved in the regulation of the axillary meristem and formation of tillers [19]. In addition, it was found that SCL3 control of GA homeostasis during root development is crucial. The SCR and SHR are two different sub-families, but both generally participate in the growth of root radial tissue by forming SCR/SHR complexes [20]. NSP1 and NSP2 genes regulate the expression of the tumor factor and participate in biosynthesis of strigolactone [21]. Moreover, the SCL4/7 sub-family can increase plant tolerance to salt and drought stress [22]. The main functions of the DLT genes are to participate in the Brazilianno steroid signal in rice and to change the induced dwarfization and low tillage phenomena [23]. According to reports, the RAM1 sub-family is involved in transduction of the root signal [16]. The RAD1 and SCL32 genes are essential for inducing upon mycorrhization [3,24]. Meanwhile, the MIG1 proteins participates in regulating the change of cellular morphology during the formation of plexus branches [25]. However, for SCLA sub-families, their functions have not been discovered yet. The DELLA protein is a repressor which participates in the gibberellin signal response [26]. GA is a plant growth regulator which can promote the growth and development of plants and has important biological functions [27].

Hence, the study of the GA biosynthesis pathway and signal transduction is a hot research topic at present. Although these GRAS families have functional characteristics in the model plants, idiographic functions of a large number of GRAS proteins have not been found.

In this study, the PgGRAS gene family was identified from the Jilin ginseng transcriptome database and studied from aspects of evolution, function and expression to further understand the important role of the PgGRAS family. Furthermore, tissue-specific expression patterns in Panax ginseng was investigated by qPCR (Real-time quantitative PCR), and the expression patterns of gibberellin acid (GA) treatment were detected. Since our study demonstrates that $\operatorname{Pg} G R A S$ gene family participates in plant secondary 
metabolism, fundamental information for the further study on plant responses to GA is provided, and the role of the GRAS gene family in the Gibberellin regulation pathway has been further verified.

\section{Materials and Methods}

\subsection{Identification of GRAS Domain Genes in the Whole Transcriptome}

The newest HMM model for the GRAS transcription factor gene family (PF03514.11) was downloaded from the Pfam database (http://pfam.sanger.ac.uk/) [28]. Screening was performed in the Jilin ginseng transcriptome using HMMER 3.0 [29]. The Jilin Ginseng database used in this experiment is composed of 248,993 transcripts extracted from 14 tissues of 4-year-old ginseng [30,31]. Then, we analyzed the amino acid sequences using NCBI CD-Search (http://www.ncbi.nlm.nih.gov/Structure/ cdd/wrpsb.cgi). Only sequences with the GRAS domain were thought to be the PgGRAS genes for the next analysis. In order to further understand the properties of PgGRAS proteins, we used Protparam (http://web.expasy.org/protparam/) to predict and analyze the chemical and physical properties of PgGRAS proteins [32].

\subsection{Conserved Motifs Analysis and Evolutionary Analysis of GRAS Genes}

We used the NCBI ORF Finder (http://www.ncbi.nlm.nih.gov/orffinder/) to find the GRAS genes with complete conserved domains. We made use of MEME (http://meme.nbcr.net/meme/) for conserved motif analysis [33]. We downloaded the sequence of the OsGRAS domain protein from the Oryza sativa genome database (http://rice.plantbiology.msu.edu/analyses_search_locus.shtml), the sequence of SIGRAS domain proteins from the Solanum lycopersicum genome database (http://solgenomics.net/ search/locus) and the AtGRAS gene sequence from the Arabidopsis thaliana genome database (http: //planttfdb.cbi.pku.edu.cn/). We constructed gene trees using the Maximum-Likelihood (ML) method of MEGA 7.0 (http://mega.co.nz/) software with 100 bootstrap replications [34]. The final gene tree was edited using the Evolview version 3.0 online web (https://www.evolgenius.info/evolview/\#login) [35].

\subsection{Gene Expression Pattern, GO Function Classification and Enrichment Analysis}

In order to understand the expression pattern of genes, the expression patterns of genes were further analyzed. The heatmaps were constructed using the TBtools version 0.6673 software [36]. Blast2go (https://www.blast2go.com/free-b2g-trial) software was used to annotate PgGRAS transcription functions [37]. Then, Gene Ontology (GO) was used for further analysis of the annotation results. The enrichment of the number of PgGRAS transcriptions classified into each subcategory was tested by the Chi-square test method.

\subsection{Network Analysis of PgGRAS Transcripts Genes}

The R programming language and software (http:/www.rproje ct.org/) was used to calculate the correlation coefficient of Spearman. A gene co-expression network was constructed using BioLayout Express $^{3 \mathrm{D}}$ version 3.2 software [38].

\subsection{Response of PgGRAS Genes to Different Concentrations of GA}

Ginseng hairy roots were cultured in 1/2 MS liquid medium for 33 days with no hormones added. Then, we obtained the secondary generation to cultivate as $1.0 \mathrm{~g}$ ginseng hairy root in 1/2 MS liquid medium each bottle. The GA was added after 23 days of culture; that is, ginseng hairy roots were cultivated by six gradients of GA concentration, which included $0.0 \mu \mathrm{M}$ as control, 2.5, 5.0, 7.5, 10.0 and $15.0 \mu \mathrm{M}$ (four repetitions per concentration gradient) [39]. At each treatment, each group of treatments was repeated three times biologically. The result is three duplicate averages, and all processing experiments were carried out in separate periods of time. The fresh weight and dry weight of the hairy roots were weighed after 30 days with the untreated hairy root as the control. All these samples were immediately frozen in liquid nitrogen and stored at $-80^{\circ} \mathrm{C}$ until RNA extraction [40]. 
For qPCR analyses, the total RNA extraction of ginseng hairy roots used the improved TRIzol method based on the published manufacturer's instructions. The quality and concentration of each RNA sample was determined using gel electrophoresis and the ScanDrop 2000 spectrophotometer (AJ, Germany). First-strand cDNA synthesis was performed using $2.00 \mu \mathrm{g}$ of total RNA in a $20 \mu \mathrm{L}$ reaction volume, according to the manufacturer's instructions for RNA purification with HiFiScript gDNA Removal cDNA Synthesis Kit (ComWin, Beijing, China). The GADPH (GenBank Accession No. KF699323.1) [41] of Panax ginseng was used as a reference gene. The qPCR of relative fluorescence quantification was measured using the Applied Biosystems 7500 Real-Time System (ABI, USA) and the Ultra SYBR Mixture Kit (Low ROX) (ComWin, Beijing, China). The designed primers (Table 1) were used to detect the response of the PgGRAS genes under GA treatment using SYBR qPCR analysis. The relative expression of the $4 P g G R A S$ genes were determined by the $2^{-\Delta \Delta C t}$ method [42] and all the samples of biological replicates and technical replicates were repeated three times.

Table 1. Sequences of $P g G R A S$ and GADPH gene primers used in this study.

\begin{tabular}{ccc}
\hline Gene Name & Forward Primer $\left(\mathbf{5}^{\prime} \mathbf{- 3}^{\prime} \mathbf{)}\right.$ & Reverse Primer $\left(\mathbf{5}^{\prime} \mathbf{3}^{\prime} \mathbf{)}\right.$ \\
\hline$P g G R A S 44-04$ & GGCAATAGAAATGGGAATAAGCGAA & CTCTTGAGAATCGACAAGTACCAAC \\
PgGRAS48-01 & TCGGACCTCAGTTACGTTGAC & TTCCTCCATCGCGGTTACAAC \\
PgGRAS50-01 & CTGCACAAATATAGCACCACCG & ATTACCCCTTCCGACCAGAATG \\
PgGRAS68-01 & GTGGAGGAACGGGTGATTGA & TCCGGCAGAGTCGTCTACTA \\
GADPH & GAGAAGGAATACACACCTGACC & CAGTAGTCATAAGCCCCTCAAC \\
\hline
\end{tabular}

\section{Results}

\subsection{Transcriptome-Wide Identification GRAS Gene Family in Ginseng}

Although the GRAS gene family has been investigated in many studies, it has not been specifically studied in Panax ginseng. In order to ensure the accuracy of the extracted sequence, two methods were used to find the sequence in this experiment. Using the GRAS, the conserved domain (PF03514) has been investigated as the inquiry sequence to search PgGRAS genes by the Hmmer method [29]. In another way, the latest Hmm (PF03514.11) of the GRAS domain was used as a blast inquiry sequence to search directly in the Jilin ginseng Transcriptome Database [30,31]. Exercising these two methods, we searched the Jilin Ginseng Transcriptome Library and finally got 131 transcripts derivatives under 79 gene IDs (Tables S1 and S2). Only 59 of these transcripts' derivatives were intact, of which five PgGRAS genes pertained to the DELLA sub-family, namely PgGRAS44-04, PgGRAS48-01, PgGRAS50-01, PgGRAS68-01 and PgGRAS19-01 of the PgGRAS genes in ginseng [43,44].

The transcripts' sequence lengths of the PgGRAS genes are between 201 (PgGRAS15-01) to 3343 bp (PgGRAS65-02). Through the analysis of the physical and chemical properties of PgGRAS proteins, we found that the molecular weights ranged from 431.00 to $87,107.53$ Daltons (Da). There are also significant differences in the theoretical PI values of predictions. The maximum theoretical PI value is 11.22 (PgGRAS75-01) and the minimum theoretical PI value is 4.37 (PgGRAS02-01). The hydrophilic range of PgGRAS proteins are from -0.652 (PgGRAS69-04) to 0.684 (PgGRAS66-06) (Table 2). Only 12 PgGRAS proteins have hydrophilicity greater than 0 , so it can be inferred that most PgGRAS proteins are hydrophilic. There were 101 PgGRAS proteins in which the number of positive amino acids was higher than that of negative amino acids, while in 25 PgGRAS proteins, the content of negative amino acids was higher than that of positive amino acids. Additionally, there were five PgGRAS proteins in which the number of positive amino acids was equal to the number of negative amino acids. It is speculated that the number of positive amino acids is greater than the number of negative amino acids in most of PgGRAS proteins. The data observation shows that most PgGRAS proteins are stable, while there are still 22 unstable PgGRAS proteins, for the reason that its instability index is less than 40 . Based on these results, it could be concluded that there are great differences in physical and chemical properties among PgGRAS proteins (Table 2). 
Table 2. Physical and chemical properties of PgGRAS 131 genes in this study.

\begin{tabular}{|c|c|c|c|c|c|c|}
\hline Name & Transcriptome ID & $\begin{array}{l}\text { Nucleic Acid } \\
\text { Length (bp) }\end{array}$ & $\begin{array}{l}\text { Amino Acid } \\
\text { Length }\end{array}$ & $\begin{array}{c}\text { Molecular } \\
\text { Weight (Da) }\end{array}$ & PI & $\begin{array}{c}\text { Average of } \\
\text { Hydropathicity }\end{array}$ \\
\hline PgGRAS01-01 & comp10366_c0_seq1 & 1634 & 431 & 431 & 5.45 & -0.401 \\
\hline PgGRAS02-01 & comp1049478_c0_seq1 & 338 & 34 & 3869.35 & 4.37 & -0.2 \\
\hline PgGRAS03-01 & comp1105291_c0_seq1 & 259 & 51 & 5983 & 11.01 & -0.282 \\
\hline PgGRAS04-01 & comp1121404_c0_seq1 & 312 & 85 & 9609.16 & 6.57 & -0.085 \\
\hline PgGRAS05-01 & comp1387617_c0_seq1 & 239 & 58 & 6767.62 & 4.87 & -0.634 \\
\hline PgGRAS06-01 & comp1596844_c0_seq1 & 258 & 78 & 8434.46 & 5.41 & -0.145 \\
\hline PgGRAS07-01 & comp17248_c0_seq1 & 210 & 68 & 7836.98 & 10.03 & -0.35 \\
\hline PgGRAS08-01 & comp17356_c0_seq1 & 2228 & 551 & $61,673.55$ & 8.84 & -0.377 \\
\hline PgGRAS08-02 & comp17356_c0_seq2 & 2145 & 551 & $61,673.55$ & 8.84 & -0.377 \\
\hline PgGRAS09-01 & comp1839771_co_seq1 & 282 & 89 & $10,316.55$ & 4.69 & -0.321 \\
\hline PgGRAS10-01 & comp19390_c0_seq1 & 258 & 54 & 6011.81 & 6.16 & -0.369 \\
\hline PgGRAS10-02 & comp19390_c0_seq2 & 221 & 39 & 4382.96 & 6.54 & -0.292 \\
\hline PgGRAS11-01 & comp2100322_c0_seq1 & 251 & 78 & 8584.94 & 4.6 & 0.306 \\
\hline PgGRAS12-01 & comp212859_c0_seq1 & 2198 & 552 & $61,408.99$ & 4.73 & -0.291 \\
\hline PgGRAS13-01 & comp22569_c0_seq1 & 1042 & 279 & $32,057.2$ & 9.06 & -0.142 \\
\hline PgGRAS14-01 & comp2261495_c0_seq1 & 210 & 55 & 5954.67 & 5.22 & -0.255 \\
\hline PgGRAS15-01 & comp23356_c0_seq1 & 201 & 30 & 3357.14 & 10.31 & 0.023 \\
\hline PgGRAS16-01 & comp25708_c0_seq1 & 865 & 266 & $29,992.15$ & 5.74 & -0.197 \\
\hline PgGRAS17-01 & comp26247_c0_seq1 & 694 & 200 & $22,426.1$ & 4.88 & 0.31 \\
\hline PgGRAS18-01 & comp2660386_co_seq1 & 202 & 39 & 4228.04 & 7.98 & 0.667 \\
\hline PgGRAS19-01 & comp267650_c0_seq1 & 1725 & 353 & $38,544.56$ & 5.69 & -0.17 \\
\hline PgGRAS20-01 & comp33784_c0_seq1 & 240 & 74 & 8432.86 & 8.8 & -0.15 \\
\hline PgGRAS21-01 & comp37210_c0_seq1 & 692 & 120 & $14,029.28$ & 9.16 & -0.512 \\
\hline PgGRAS22-01 & comp38081_c0_seq1 & 1876 & 547 & $60,343.12$ & 5.89 & -0.362 \\
\hline PgGRAS23-01 & comp40091_c0_seq1 & 956 & 229 & $24,803.16$ & 4.85 & 0.083 \\
\hline PgGRAS24-03 & comp418104_c0_seq1 & 1633 & 437 & $49,927.34$ & 9.22 & -0.45 \\
\hline PgGRAS25-03 & comp44037_c0_seq3 & 782 & 72 & 8236.58 & 4.44 & 0.321 \\
\hline PgGRAS26-01 & comp46234_c0_seq1 & 1723 & 527 & $60,102.48$ & 4.61 & -0.422 \\
\hline PgGRAS27-01 & comp472334_c0_seq1 & 733 & 218 & $24,669.44$ & 4.52 & 0.139 \\
\hline PgGRAS28-01 & comp479040_c0_seq1 & 578 & 172 & $19,639.29$ & 9.01 & -0.61 \\
\hline PgGRAS29-01 & comp50021_c0_seq1 & 652 & 129 & $14,932.18$ & 6.19 & -0.156 \\
\hline PgGRAS30-01 & comp51501_c0_seq1 & 2097 & 523 & $59,299.2$ & 5.99 & -0.48 \\
\hline PgGRAS31-01 & comp52182_c0_seq1 & 1105 & 244 & $26,552.45$ & 4.9 & -0.454 \\
\hline PgGRAS32-01 & comp522134_c0_seq1 & 756 & 236 & $25,687.72$ & 6.37 & 0.198 \\
\hline PgGRAS33-01 & comp53859_c0_seq1 & 1411 & 358 & $59,615.92$ & 5.74 & -0.31 \\
\hline PgGRAS34-10 & comp53978_c4_seq10 & 2272 & 541 & $59,954.61$ & 5.71 & -0.249 \\
\hline PgGRAS34-02 & comp53978_c4_seq2 & 2346 & 536 & $40,253.29$ & 6.61 & -0.134 \\
\hline PgGRAS34-05 & comp53978_c4_seq5 & 2194 & 541 & $59,615.92$ & 5.74 & -0.31 \\
\hline PgGRAS35-01 & comp546436_c0_seq1 & 540 & 109 & $12,690.7$ & 8.46 & -0.376 \\
\hline PgGRAS36-01 & comp54894_c0_seq1 & 2441 & 674 & $74,618.25$ & 5.84 & -0.396 \\
\hline PgGRAS36-02 & comp54894_c0_seq2 & 915 & 198 & $22,784.94$ & & -0.339 \\
\hline PgGRAS37-01 & comp564672_c0_seq1 & 400 & 107 & $12,078.8$ & 4.73 & -0.128 \\
\hline PgGRAS38-01 & comp572133_c0_seq1 & 458 & 141 & $15,201.03$ & 5.07 & -0.227 \\
\hline PgGRAS39-01 & comp57286_c0_seq1 & 704 & 148 & $15,923.56$ & 4.53 & -0.376 \\
\hline PgGRAS40-01 & comp578948_c0_seq1 & 309 & 91 & $10,079.48$ & 11.16 & -0.552 \\
\hline PgGRAS41-04 & comp59054_c0_seq4 & 930 & 168 & $19,216.92$ & 5.11 & -0.066 \\
\hline PgGRAS42-02 & comp59054_c1_seq2 & 345 & 76 & 8355.65 & 8.19 & -0.095 \\
\hline PgGRAS43-01 & comp59228_c0_seq1 & 2069 & 564 & $62,183.25$ & 5.99 & -0.521 \\
\hline PgGRAS44-04 & comp59348_c0_seq4 & 1635 & 532 & $58,791.83$ & 4.97 & -0.15 \\
\hline PgGRAS45-01 & comp59447_c0_seq1 & 1622 & 413 & $45,985.08$ & 7.7 & -0.094 \\
\hline PgGRAS46-01 & comp61562_c0_seq1 & 2110 & 477 & $53,963.14$ & 6.05 & -0.161 \\
\hline PgGRAS47-01 & comp61661_c3_seq1 & 2420 & 542 & $60,731.41$ & 5.54 & -0.335 \\
\hline PgGRAS48-01 & comp62080_c0_seq1 & 2058 & 520 & $57,513.1$ & 5.27 & -0.128 \\
\hline PgGRAS49-01 & comp62792_c0_seq1 & 2000 & 493 & $55,553.44$ & 5.91 & -0.126 \\
\hline PgGRAS49-02 & comp62792_c0_seq2 & 2015 & 498 & $55,978.88$ & 5.95 & -0.114 \\
\hline PgGRAS49-03 & comp62792_c0_seq3 & 1385 & 375 & $42,219.01$ & 5.37 & -0.177 \\
\hline PgGRAS49-04 & comp62792_c0_seq4 & 1373 & 370 & $41,793.58$ & 5.37 & -0.194 \\
\hline PgGRAS49-05 & comp62792_c0_seq5 & 1891 & 493 & $55,612.53$ & 5.84 & -0.129 \\
\hline PgGRAS49-06 & comp62792_c0_seq6 & 1906 & 498 & $56,037.97$ & 5.87 & -0.117 \\
\hline PgGRAS50-01 & comp62989_c0_seq1 & 2491 & 580 & $63,896.36$ & 5.04 & -0.243 \\
\hline PgGRAS51-01 & comp63201_c1_seq1 & 1875 & 408 & $46,130.96$ & 6 & -0.103 \\
\hline PgGRAS51-02 & comp63201_c1_seq2 & 2487 & 608 & $67,328.55$ & 6.14 & -0.225 \\
\hline PgGRAS52-01 & comp63447_c0_seq1 & 1761 & 518 & $58,723.53$ & 4.6 & -0.275 \\
\hline PgGRAS52-02 & comp63447_c0_seq2 & 1747 & 518 & $58,723.53$ & 4.6 & -0.275 \\
\hline PgGRAS53-01 & comp63847_c0_seq1 & 2308 & 598 & $65,887.92$ & 4.88 & -0.277 \\
\hline PgGRAS54-01 & comp64175_c2_seq1 & 3053 & 798 & $86,476.95$ & 5.92 & -0.284 \\
\hline
\end{tabular}


Table 2. Cont.

\begin{tabular}{|c|c|c|c|c|c|c|}
\hline Name & Transcriptome ID & $\begin{array}{l}\text { Nucleic Acid } \\
\text { Length (bp) }\end{array}$ & $\begin{array}{l}\text { Amino Acid } \\
\text { Length }\end{array}$ & $\begin{array}{c}\text { Molecular } \\
\text { Weight (Da) }\end{array}$ & PI & $\begin{array}{c}\text { Average of } \\
\text { Hydropathicity }\end{array}$ \\
\hline PgGRAS54-02 & comp64175_c2_seq2 & 3085 & 795 & $86,246.77$ & 6 & -0.289 \\
\hline PgGRAS55-01 & comp64629_c0_seq1 & 2043 & 541 & $60,690.52$ & 6 & -0.343 \\
\hline PgGRAS55-02 & comp64629_c0_seq2 & 1746 & 470 & $52,338.84$ & 5.57 & -0.339 \\
\hline PgGRAS56-02 & comp64837_c0_seq2 & 3009 & 578 & $64,507.79$ & 5.87 & -0.421 \\
\hline PgGRAS57-01 & comp65004_c0_seq1 & 2924 & 612 & $66,846.76$ & 6.69 & -0.294 \\
\hline PgGRAS58-01 & comp65321_c0_seq1 & 916 & 143 & $16,880.14$ & 6.51 & -0.483 \\
\hline PgGRAS58-11 & comp65321_c0_seq11 & 1094 & 143 & $16,880.14$ & 6.51 & -0.483 \\
\hline PgGRAS58-13 & comp65321_c0_seq13 & 1958 & 143 & $16,880.14$ & 6.51 & -0.483 \\
\hline PgGRAS58-17 & comp65321_c0_seq17 & 1254 & 143 & $16,880.14$ & 6.51 & -0.483 \\
\hline PgGRAS58-18 & comp65321_c0_seq18 & 1760 & 143 & $16,880.14$ & 6.51 & -0.483 \\
\hline PgGRAS58-20 & comp65321_c0_seq20 & 1087 & 143 & $16,880.14$ & 6.51 & -0.483 \\
\hline PgGRAS58-03 & comp65321_c0_seq3 & 928 & 143 & $16,880.14$ & 6.51 & -0.483 \\
\hline PgGRAS58-35 & comp65321_c0_seq35 & 1123 & 143 & $16,880.14$ & 6.51 & -0.483 \\
\hline PgGRAS58-37 & comp65321_c0_seq37 & 1109 & 143 & $16,880.14$ & 6.51 & -0.483 \\
\hline PgGRAS58-04 & comp65321_c0_seq4 & 1009 & 143 & $16,880.14$ & 6.51 & -0.483 \\
\hline $\operatorname{PgGRAS58-47}$ & comp65321_c0_seq47 & 449 & 123 & $14,595.54$ & 5.93 & -0.464 \\
\hline PgGRAS59-01 & comp65321_c2_seq1 & 435 & 131 & $15,178.62$ & 9.23 & -0.41 \\
\hline PgGRAS60-01 & comp65331_c0_seq1 & 2630 & 765 & $87,107.53$ & 6.06 & -0.462 \\
\hline PgGRAS60-02 & comp65331_c0_seq2 & 2791 & 765 & $87,107.53$ & 6.06 & -0.462 \\
\hline PgGRAS61-01 & comp65403_c0_seq1 & 2066 & 575 & $64,422.68$ & 5.09 & -0.347 \\
\hline PgGRAS61-02 & comp65403_c0_seq2 & 1021 & 294 & $33,240.21$ & 6.03 & -0.14 \\
\hline PgGRAS62-01 & comp65479_c0_seq1 & 2282 & 522 & $57,819.13$ & 5.79 & -0.053 \\
\hline PgGRAS62-02 & comp65479_c0_seq2 & 2265 & 443 & $48,548.35$ & 5.63 & -0.03 \\
\hline PgGRAS62-03 & comp65479_c0_seq3 & 3176 & 546 & $60,663.43$ & 5.11 & -0.229 \\
\hline PgGRAS62-04 & comp65479_c0_seq4 & 2137 & 443 & $48,548.35$ & 5.63 & -0.03 \\
\hline PgGRAS62-05 & comp65479_c0_seq5 & 3135 & 546 & $60,663.43$ & 5.11 & -0.229 \\
\hline PgGRAS62-06 & comp65479_c0_seq6 & 3025 & 380 & $42,404.63$ & 5.49 & -0.03 \\
\hline PgGRAS62-07 & comp65479_c0_seq7 & 2984 & 380 & $42,404.63$ & 5.49 & -0.03 \\
\hline PgGRAS62-08 & comp65479_c0_seq8 & 2154 & 522 & $57,819.13$ & 5.79 & -0.053 \\
\hline PgGRAS63-01 & comp66169_c0_seq1 & 3313 & 754 & $84,839.24$ & 5.61 & -0.446 \\
\hline PgGRAS63-02 & comp66169_c0_seq2 & 2999 & 754 & $84,839.24$ & 5.16 & -0.446 \\
\hline PgGRAS64-01 & comp66380_c0_seq1 & 2230 & 470 & $52,786.49$ & 5.91 & -0.185 \\
\hline PgGRAS64-02 & comp66380_c0_seq2 & 2291 & 465 & $52,285.89$ & 5.94 & -0.221 \\
\hline PgGRAS64-06 & comp66380_c0_seq6 & 2305 & 465 & $52,285.89$ & 5.94 & -0.221 \\
\hline PgGRAS64-08 & comp66380_c0_seq8 & 1013 & 317 & $35,871.94$ & 6.17 & -0.38 \\
\hline PgGRAS64-09 & comp66380_c0_seq9 & 2259 & 470 & $52,786.49$ & 5.91 & -0.185 \\
\hline PgGRAS65-01 & comp67093_c0_seq1 & 2855 & 753 & $84,264.99$ & 5.32 & -0.437 \\
\hline PgGRAS65-02 & comp67093_c0_seq2 & 3343 & 753 & $84,264.99$ & 5.32 & -0.437 \\
\hline PgGRAS65-03 & comp67093_c0_seq3 & 2904 & 753 & $84,264.99$ & 5.32 & -0.437 \\
\hline PgGRAS65-04 & comp67093_c0_seq4 & 3054 & 753 & $84,264.99$ & 5.32 & -0.437 \\
\hline PgGRAS66-02 & comp67249_c0_seq2 & 498 & 75 & 8053.39 & 9.22 & 0.505 \\
\hline PgGRAS66-06 & comp67249_c0_seq6 & 484 & 50 & 5415.45 & 9.4 & 0.684 \\
\hline PgGRAS67-12 & comp67428_c0_seq12 & 1917 & 569 & $63,367.08$ & 5.03 & -0.123 \\
\hline PgGRAS67-25 & comp67428_c0_seq25 & 1299 & 349 & $39,076.4$ & 6.35 & 0.106 \\
\hline PgGRAS67-26 & comp67428_c0_seq26 & 1891 & 569 & $63,367.08$ & 5.04 & -0.123 \\
\hline PgGRAS68-01 & comp67501_c0_seq1 & 2041 & 540 & $59,906.01$ & 5.03 & -0.188 \\
\hline PgGRAS68-02 & comp67501_c0_seq2 & 1733 & 361 & $39,883.54$ & 5.83 & -0.057 \\
\hline PgGRAS69-01 & comp67516_c0_seq2 & 2051 & 499 & $58,454.58$ & 8.26 & -0.651 \\
\hline PgGRAS69-02 & comp67516_c0_seq3 & 2633 & 726 & $83,814.41$ & 5.91 & -0.623 \\
\hline PgGRAS69-03 & comp67516_c0_seq4 & 2099 & 499 & $58,454.58$ & 8.26 & -0.651 \\
\hline PgGRAS69-04 & comp67516_c0_seq6 & 2538 & 671 & $77,840.89$ & 6.02 & -0.652 \\
\hline PgGRAS70-01 & comp67518_c0_seq1 & 1172 & 123 & $14,161.05$ & 6.28 & -0.407 \\
\hline PgGRAS70-02 & comp67518_c0_seq2 & 1081 & 129 & $15,078.18$ & 7.63 & -0.416 \\
\hline PgGRAS70-03 & comp67518_c0_seq3 & 1415 & 123 & $14,161.05$ & 6.28 & -0.407 \\
\hline PgGRAS70-04 & comp67518_c0_seq4 & 1055 & 129 & $15,078.18$ & 7.63 & -0.416 \\
\hline PgGRAS70-08 & comp67518_c0_seq8 & 1298 & 129 & $15,078.18$ & 7.63 & -0.416 \\
\hline PgGRAS71-01 & comp708716_c0_seq1 & 492 & 86 & 9590.63 & 5.49 & -0.316 \\
\hline PgGRAS72-01 & comp726689_c0_seq1 & 415 & 119 & $12,725.35$ & 5.31 & -0.224 \\
\hline PgGRAS73-01 & comp753553_c0_seq1 & 363 & 78 & 7635.45 & 6.04 & 0.026 \\
\hline PgGRAS74-01 & comp762664_c0_seq1 & 618 & 192 & $23,111.62$ & 8.81 & -0.505 \\
\hline PgGRAS75-01 & comp774347_c0_seq1 & 439 & 60 & 7033.28 & 11.22 & -0.522 \\
\hline PgGRAS76-01 & comp866028_c0_seq1 & 430 & 68 & 7436.13 & 6.93 & -0.503 \\
\hline PgGRAS77-01 & comp876245_c0_seq1 & 1036 & 209 & $23,803.37$ & 6.09 & -0.293 \\
\hline PgGRAS78-01 & comp913576_c0_seq1 & 443 & 58 & 6237.18 & 5.4 & -0.084 \\
\hline PgGRAS79-01 & comp933760_c0_seq1 & 420 & 132 & $14,868.61$ & 5.93 & -0.413 \\
\hline
\end{tabular}




\subsection{Conserved Motif Analysis and Systematic Analysis of PgGRAS Gene Family}

By analyzing these results, we have obtained 131 transcripts' sequences of GRAS genes in ginseng. For the sequence-structure study, we used the NCBI ORF Finder to search for the ORFs of 131 transcripts. We found that 59 transcripts had a complete ORF. However, the GRAS gene family has a unique conserved domain, in addition to other motifs. Additionally, there are five main motifs in conserved domains. The GRAS gene family has 20 motifs found throughout the literature (Figure 1a) [45]. Applying MEME for the analysis of these conserved motifs, we found that most of the transcripts have similar conserved motifs. The stability of the conserved domain is proven in Figure $1 \mathrm{~b}$.

(a)

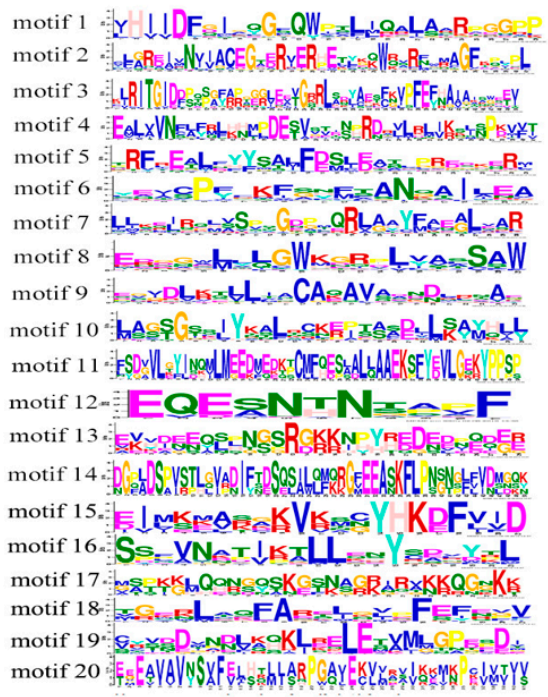

(b)

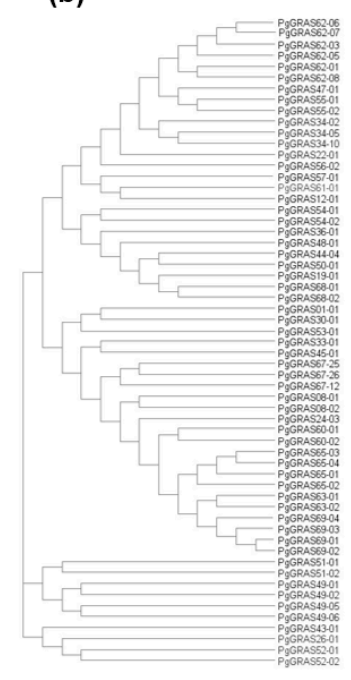

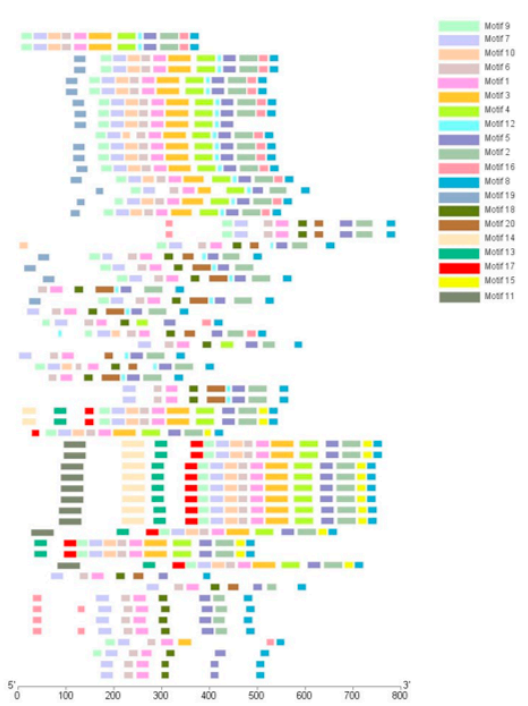

Figure 1. Analysis of conserved domains of PgGRAS proteins. (a) Conserved domains inferred from PgGRAS protein sequences are highlighted by MEME software. (b) Maximum-likelihood tree of PgGRAS proteins is shown on the left. Simultaneously display different subfamilies with different colors. Using different color motifs to reveal the conserved domain of the corresponding protein on the right.

In order to help with $P g G R A S$ classification and decipher the evolution history of the $\operatorname{Pg} G R A S$ gene family in ginseng, we built two evolutionary trees and applied 67 genes (Ginseng and Arabidopsis) (Figure 2a; Tables S2 and S3) and 172 genes (Ginseng, Arabidopsis, Tomato and Rice) (Figure 2b; Tables S2 and S3), respectively [46,47]. As shown in Figure 2, we identified a total of 15 sub-families of the GRAS genes. For ginseng, we identified nine PgGRAS genes in the PAT sub-families, six in the LISCL sub-family, five in the DELLA sub-families, two in the HAM sub-family, two in the SHR sub-family, two in the NSP2 sub-families, one in the SCL3 sub-family, one in the DLT sub-family, one in the NSP1 sub-families, one in the SCL4/7 sub-families, one in the RAM1 sub-family, one in the SCR sub-family and one in the PG1 sub-family (Figure 2a). The LLSCL sub-family has 13 members, and six PgGRAS genes (PgGRAS08-1, PgGRAS24-03, PgGRAS60-01, PgGRAS63-01, PgGRAS65-01 and PgGRAS69-01) are highly homologous to AtSCL9 (AtGRAS27) and may be associated with the regulation of the occurrence of microspores in meiosis [48,49]. The PAT sub-family has 15 members, nine of which (PgGRAS12-01, PgGRAS22-01, PgGRAS34-02, PgGRAS47-01, PgGRAS55-01, PgGRAS56-02, PgGRAS57-01, PgGRAS61-01 and PgGRAS62-01) have a high degree of similarity to AtPAT (AtGRAS30) [50]. Based on previous studies, it was found that the Arabidopsis GRAS protein PAT was involved in the early stage of photosensitive pigment a signal transduction. These nine members may have similar functions. It has been reported that the HAM and LS families are involved in the development and regulation of axillary birth tissue, the apex meristem and leaf axils meristem of tiller formation, respectively [49]. The HAM sub-family consists of six members (PgGRAS49-01, PgGRAS51-01,AtGRAS15, AtGRAS22, AtGRAS23 and AtGRAS26) and the LS sub-family consists of 
one member (AtGRAS7). The SCR and SHR sub-families are critical to the growth of radial tissue during the development of roots and buds. A total of three members (PgGRAS01-01,PgGRAS30-01 and PgGRAS54-01) of the GRAS gene family belong to the SCR and SHR families and may be associated with the development of roots and buds [48].
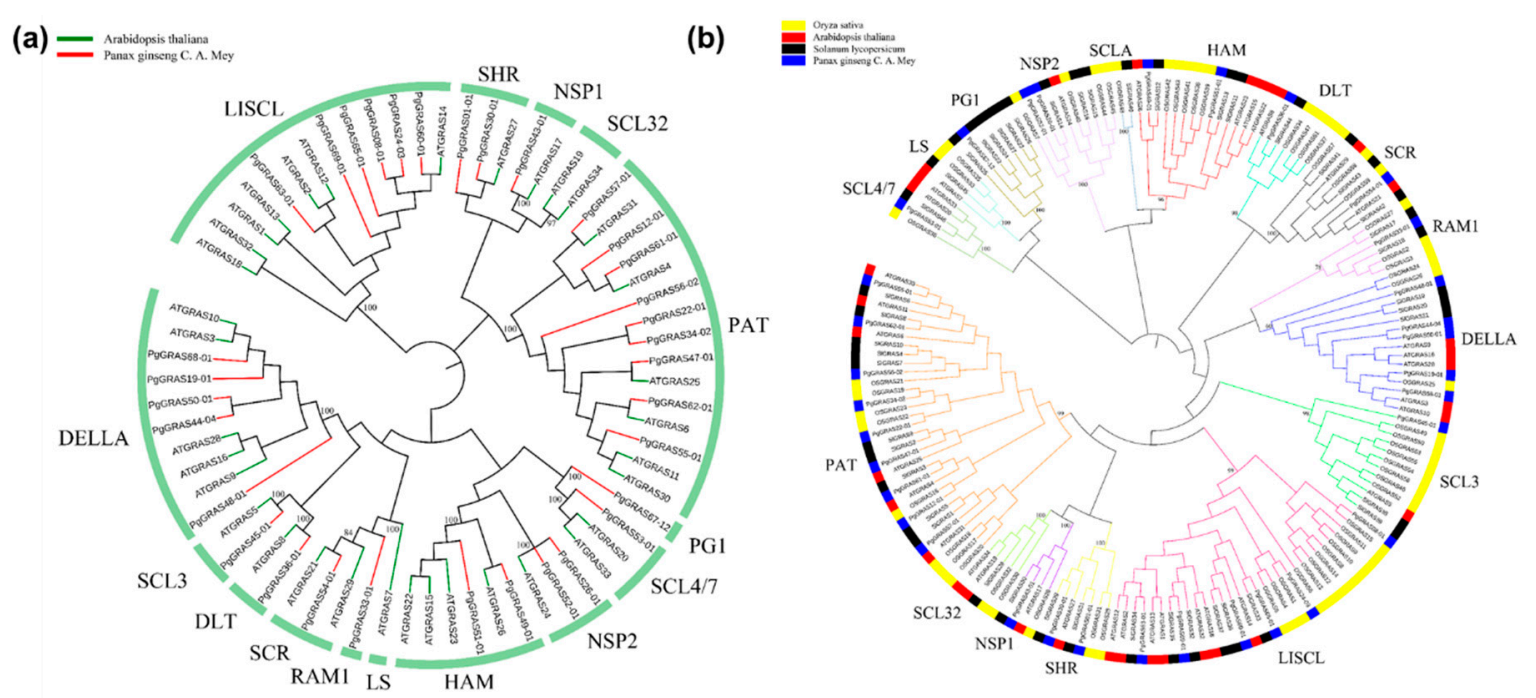

Figure 2. Evolutionary tree of the PgGRAS protein. (a) PgGRAS proteins were divided into 15 subfamilies according to the classification of Arabidopsis thaliana by using the maximum-likelihood (ML) method. Green, Arabidopsis thaliana. Red, Panax ginseng C. A. Meyer. (b) Evolutionary analysis of GRAS proteins in using the maximum-likelihood (ML) method to construct an evolutionary tree of Solanum lycopersicum, Panax ginseng C. A. Meyer, Oryza sativa and Arabidopsis thaliana. Members with the same color of the clade belong to the same subfamily. Members with the same color on the outer circle are of the same species. Black, Solanum lycopersicum. Red, Arabidopsis thaliana. Yellow, Oryza sativa. Blue, Panax ginseng C. A. Meyer.

The RAM1 sub-family contains only one ginseng gene (PgGRAS33-01), and the RAM1 protein is involved in inducing many AM (Arbuscular mycorrhiza)-related genes. Through the above, we can boldly infer that the GRAS gene is likely to be involved in the related process of AM. The SCL3 sub-family has two members (AtGRAS5 and PgGRAS45-01) and one member in ginseng.

The DLT genes were involved in the brassinosteroid (BR) signaling, but only one gene (PgGRAS36-01) belongs to the DLT sub-family in ginseng [51]. NSP1 and NSP2 regulate the expression of genes associated with strigolactone biosynthesis, so that the root can be stimulated to mycorrhization by affecting hormones and their derivatives [3]. After analysis, it was found that three ginseng genes (PgGRAS26-01, PgGRAS43-01 and PgGRAS52-01) belong to the NSP1 and NSP2 sub-families. Three genes (AtGRAS20, AtGRAS33 and PgGRAS53-01) were observed in the evolutionary tree belonging to the SCL4/7 subfamily, which may be involved in the resistance system in the plant [2]. However, the SCL32 subfamily contains only two members (AtGRAS19 and AtGRAS34) of the Arabidopsis thaliana, and there are no members of the SCL32 sub-family in the Panax ginseng. There was a unique subfamily found, namely PG1 (PgGRAS67-12), in ginseng (Figure 2a). As is known, the GRAS gene family has a subfamily DELLA that is involved in gibberellin signal responses and steady-state balance; the sub-family DELLA consists of 10 members (PgGRAS68-01,PgGRAS19-01, PgGRAS50-01, PgGRAS44-04, PgGRAS48-01, AtGRAS10, AtGRAS3, AtGRAS28, AtGRAS16 and AtGRAS9), among which five members are from ginseng (Figure 2a). By understanding the DELLA sub-family, we can further study its influence on GA and speculate on its role in GA pathway [52].

According to the ML evolutionary tree, it can be seen that the genes of Panax ginseng, Arabidopsis thaliana, Solanum lycopersicum and Oryza sativa are mainly distributed in 13 sub-families (Figure 2b). 
No genes of ginseng were found in the SCLB sub-family. These results suggest that the origin of the $\operatorname{PgGRAS}$ gene family was before the separation of monocotyledons and dicotyledons [9,53].

\subsection{Network Analysis of Gene Expression in PgGRAS Gene Family}

By means of the analysis of gene family genes, it can be said that there are great differences in expression and differentiation among functions of the family members of the gene. In order to observe the network of interactions among genes, we selected 131 transcripts expressed in 14 tissues from 42 farmers' ginseng cultivars. Figure 3 shows a network of expression interactions among 131 transcripts (Figure 3a). These results show that with the functional differentiation of the PgGRAS gene family, the expression of its gene members is also different [54]. This phenomenon is not only observed within the gene family, but also sub-families. For the sake of checking whether the interaction among genes is associated with expression activities to some extent, we randomly sampled as the negative control the transcripts of 14 tissues of 4-year-old ginseng. Then, $131 \mathrm{PgGRAS}$ transcripts tend to have associated expression and form a co-expression network (Figure $3 b$ ). These results show that the function and expression of the gene members of the $P g G R A S$ gene family have been significantly differentiated. However, they do maintain a slight link between relevant expression and functional cooperation [55].

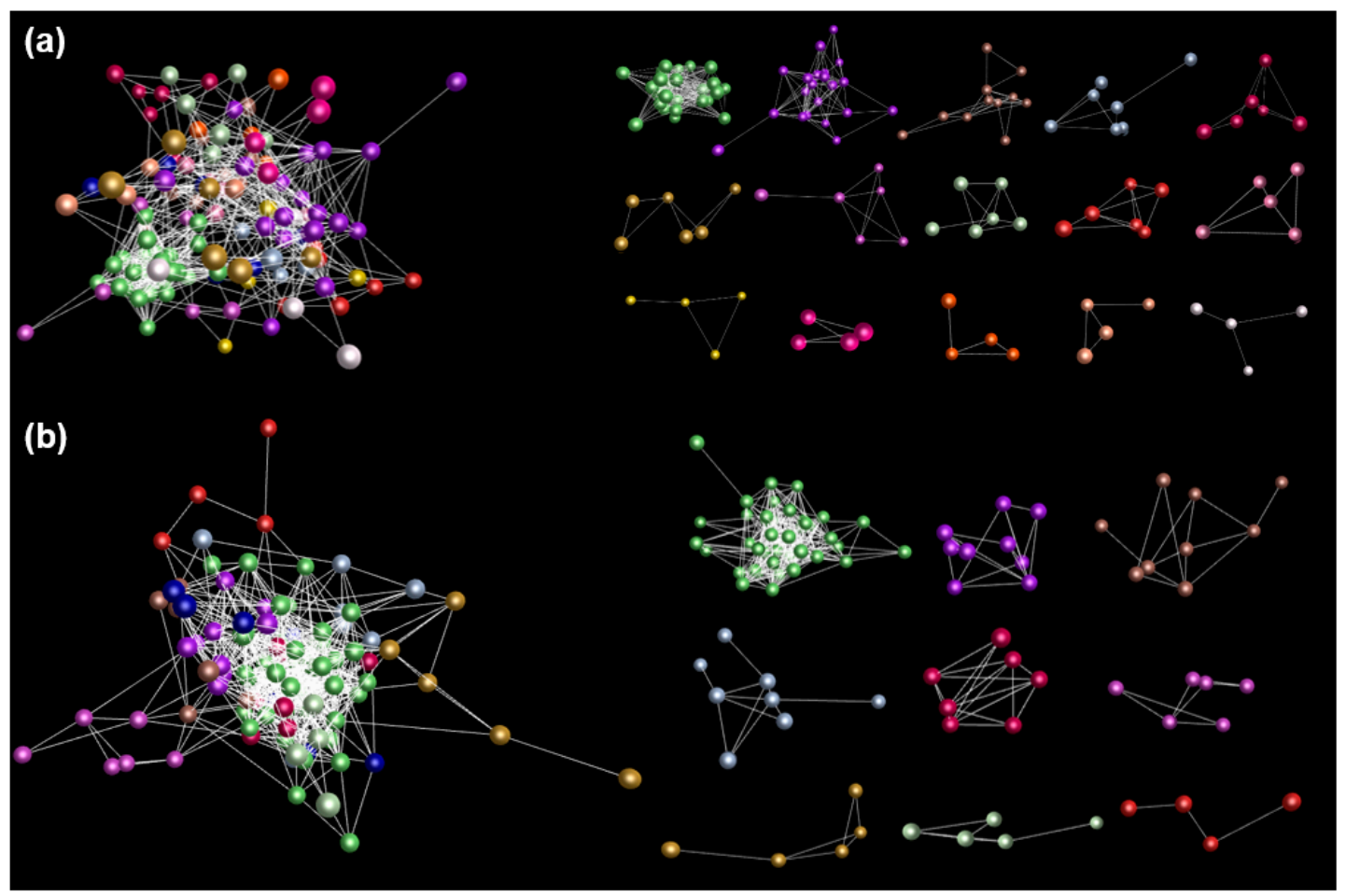

Figure 3. Network of the $P g G R A S$ genes of the PgGRAS gene family. (a) Functional analysis of $P g G R A S$ genes in 14 tissues from a 4-year-old ginseng plant. (b) Functional analysis of PgGRAS genes in 4-year-old ginseng roots of 42 ginseng farmers' cultivars. Different color balls indicate the PgGRAS genes selected from different clusters of the $\operatorname{Pg} G R A S$ gene family.

\subsection{Expression Analysis and Functional Evolution of PgGRAS Gene Family}

In order to further understand the expression of PgGRAS genes in ginseng, we used the selection of 131 ginseng transcripts for analysis: we analyzed the expressions of 131 transcripts and drew the heatmaps in the roots of 5-, 12-, 18- and 25-year-old ginseng plants. Fourteen tissues samples were taken from a 4-year-old ginseng plant; the 4-year-old roots of 42 genotypes were collected from 42 farmers and the expression profiles (Figure $4 \mathrm{a}-\mathrm{c}$ and Table S4). At least 85 transcription profiles were found in 131 transcripts. At least 85 of 131 transcripts were expressed, and a number of PgGRAS genes had similar expression patterns. Heatmaps indicated that lots of $P g G R A S$ genes not only had similar 
expression patterns, but also interactions. It is further shown that there is a network of interactions among genes to control gene expression. In order to improve the understanding of the functional relationships among gene sub-families, we used the Gene Ontology (GO) terms to analyze gene functions. According to the results, we found that 131 transcripts are divided into three main GO function categories-Biological Processes (BP), Molecular Function (MF) and Cellular Components (CC) [56]. Most of these transcripts have three functions at the same time, with only 15 genes having a single function (Figure 5a). These three terms have eight subcategories in Level 2-BP includes cellular process, biological regulation, metabolic process, signaling, multicellular organismal process, regulation of biological process, response to stimulus, developmental process and single-organism process; MF includes transcription factor activity, protein binding, binding and nucleic acid binding transcription factor activity; CC includes cell parts, cells and organelles. For more information about this study, the enrichment analysis was carried out. An enrichment analysis revealed that 11 subcategories in 15 subcategories were significantly enriched $(p \leq 0.01)$ in comparison with total transcriptome transcripts (Figure 6). Then, we performed a functional analysis of $131 \mathrm{Pg} G R A S$ genes that were found to have intact conserved domains (Figure $5 b-d$ and Table S5). These results indicate that the gene function of each type of $\mathrm{PgGRAS}$ family had differentiated as they evolved.

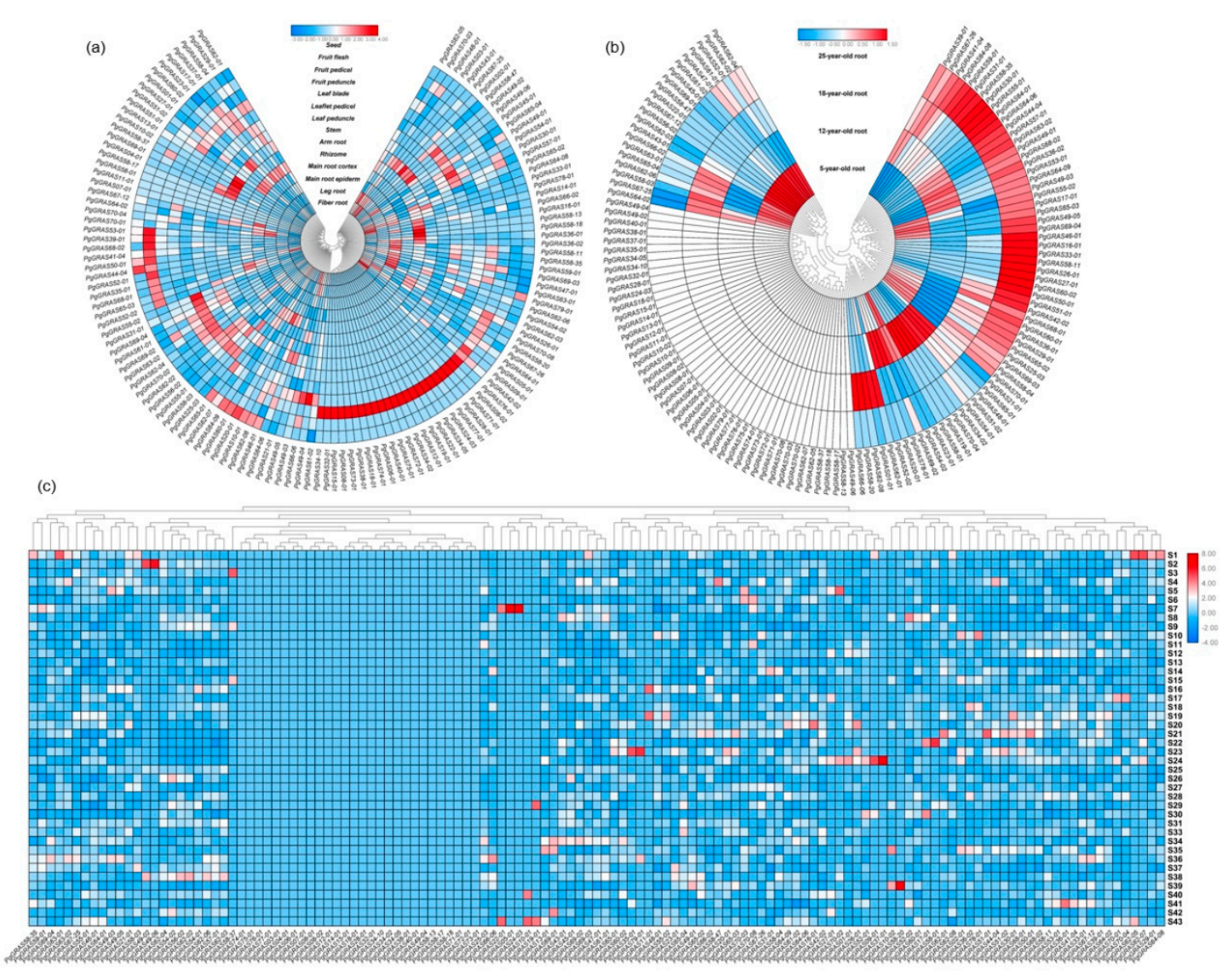

Figure 4. Relationship of the $P g G R A S$ gene with their expression activities. (a) The expression spectrums of the 131 PgGRAS transcripts in 14 tissues of a 4-year-old ginseng plant. (b) The expression spectrums of the $131 \mathrm{PgGRAS}$ transcripts in 5-, 12-, 18- and 25-year-old ginseng plants. (c) The expression spectrums of the $131 \mathrm{PgGRAS}$ genes in 4-year-old roots of 42 ginseng farmers' cultivars. The shades of color represent a relatively higher or lower level of expression. 

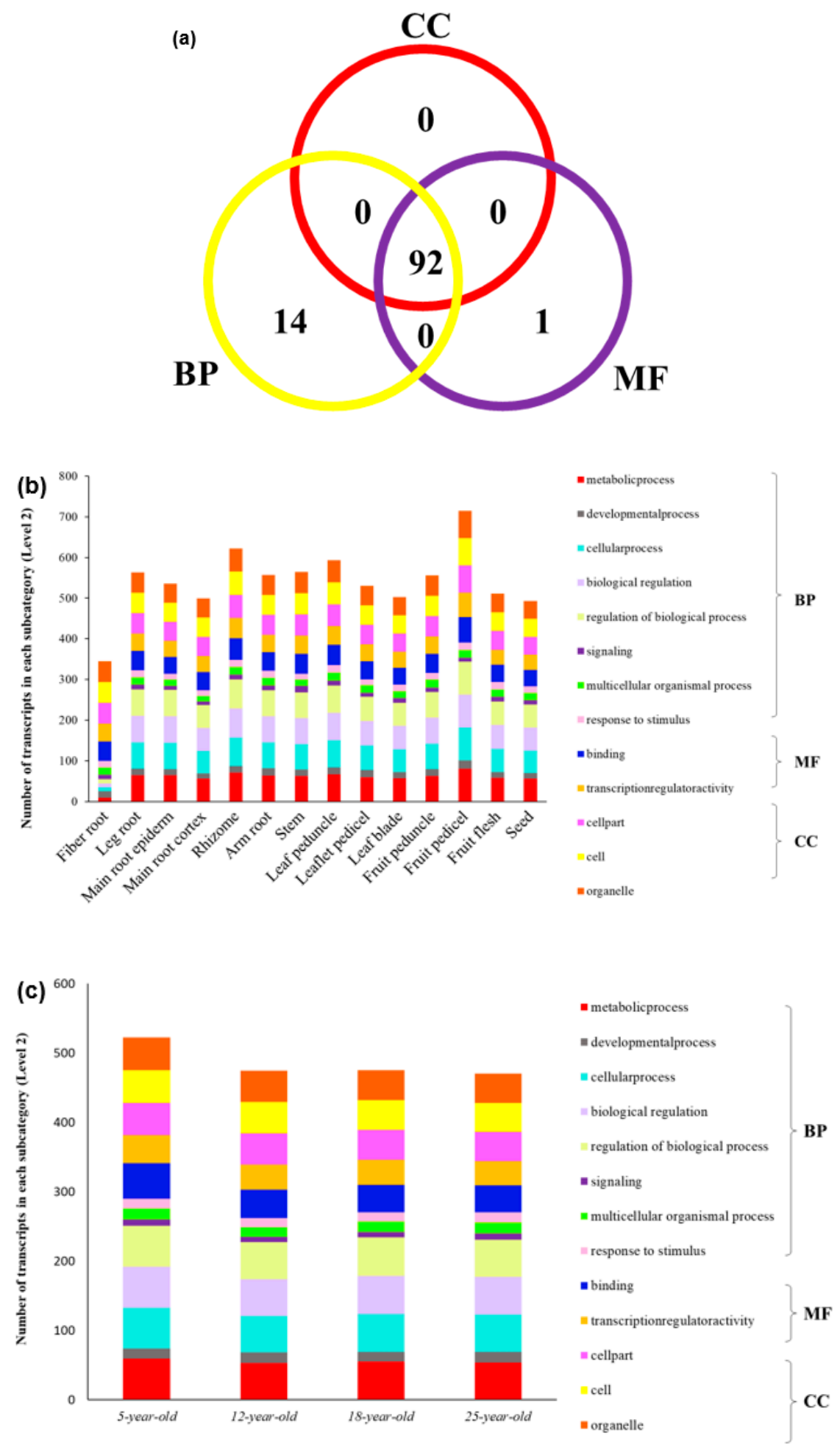

Figure 5. Cont. 


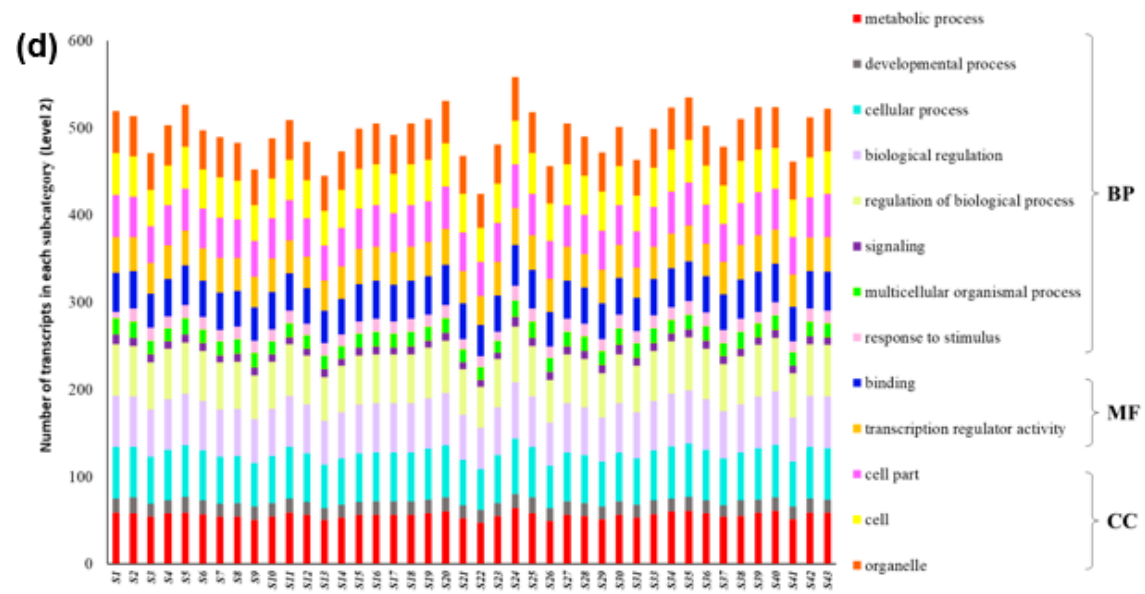

Figure 5. Functional categorization of the PgGRAS gene transcripts by Gene Ontology (GO). (a) Venn diagram of the functional categorization of the PgGRAS transcripts. Biological processes (BP), molecular function (MF) and cellular components (CC). (b) Variation of the functional categories of the PgGRAS transcripts among 14 tissues of a 4-year-old ginseng plant. (c) Variation of the functional categories of the PgGRAS transcripts among 5-, 12-, 18- and 25-year-old ginseng plants. (d) Variation of the functional categories of the PgGRAS transcripts among 4-year-old roots of 42 ginseng farmers' cultivars.

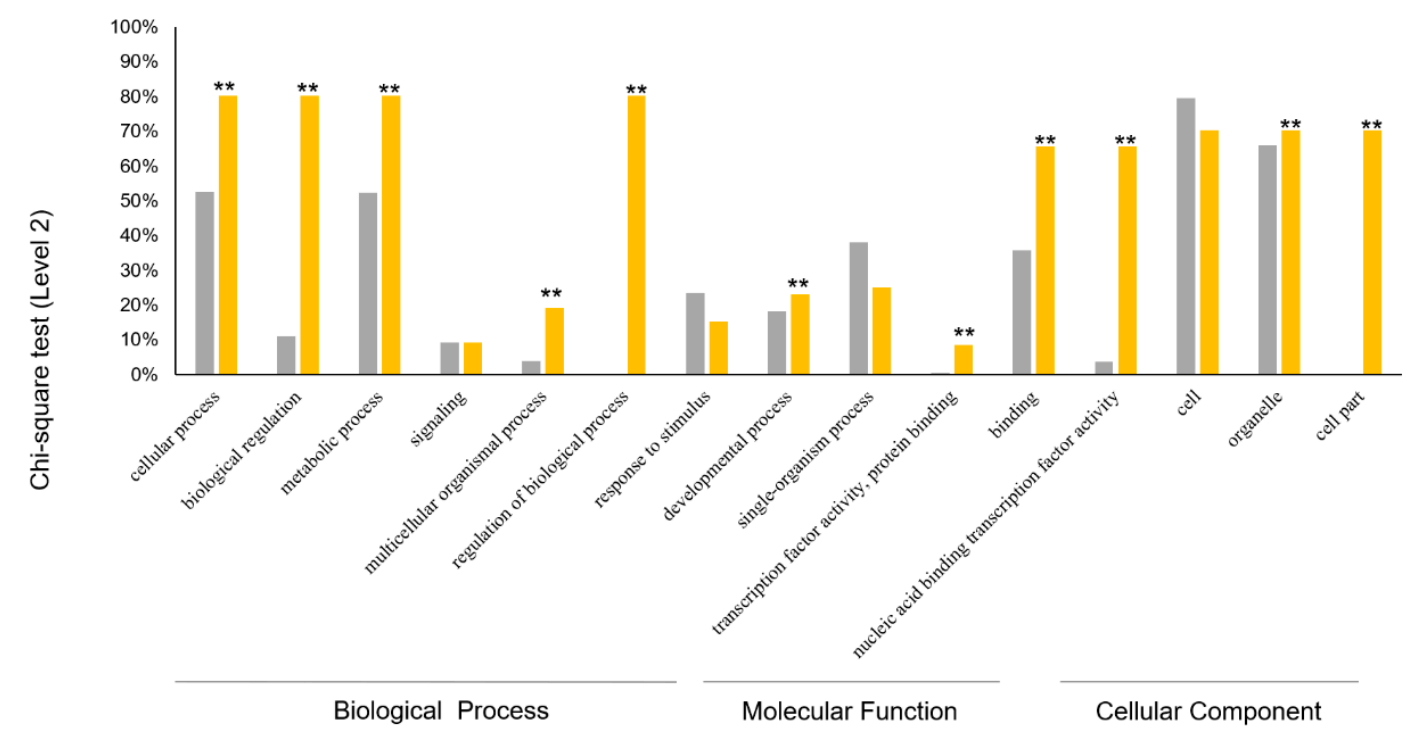

Figure 6. The Chi-square test was used to calculate the prediction and actual situation of the same function (Level 2) of the PgGRAS genes, further embodying the differentiation diversity of the PgGRAS genes function. Gray, the control group. Yellow, the $\operatorname{Pg} G R A S$ genes group. ${ }^{* *}$, very significant $(p \leq 0.01)$.

\subsection{Analysis of PgGRAS Genes Expression under Different Concentrations of GA}

Plant hormones play an important role in the growth and development of plants, and GRAS transcription factors play an important role in the pathogenesis of metabolism and biosynthesis in plant growth $[57,58]$. Firstly, the growth and development of ginseng hairy roots treated with different concentrations of GA were measured. The fresh weight and dry weight of all the treatments reached their peak at the GA concentration of $10 \mu \mathrm{M}$, but were still less than those of control group (Figure 7). GRAS transcription factors are also involved in plant growth metabolic and biosynthetic pathways. After previous analysis, it was identified that PgGRAS44-04, PgGRAS48-01, PgGRAS50-01, PgGRAS68-01 and PgGARS19-01 belong to the DELLA sub-family, thus, it is speculated that it has the function of responding to gibberellin. After detection, the expression levels of these five genes were different under different concentrations of GA treatment. PgGARS19-01 was not detected in the qPCR assay. Hence, no further analysis was performed. Here, we found that three genes-PgGRAS44-04, 
PgGRAS48-01 and PgGRAS50-01-all reached their peaks of expression at $10.0 \mu \mathrm{M}$ among treatments with different concentrations of GA (Figure $8 \mathrm{a}-\mathrm{c}$ ). The expressions of these three genes were gradually upregulated until GA reached the concentration of $10.0 \mu \mathrm{M}$, from which the expression levels decreased (Figure 8a-c). The expression level of $P g G R A S 68-01$ began to gradually decline after the concentration of $7.5 \mu \mathrm{M}$ (Figure 8d). The results show that PgGRAS44-04, PgGRAS48-01 and PgGRAS50-01 had similar expression patterns. PgGRAS44-04, PgGRAS48-01, PgGRAS50-01 and PgGRAS68-01 all had different responses to exogenous GA addition. It is further demonstrated that the PgGRAS gene family can be applied to GA stress response.

(a)

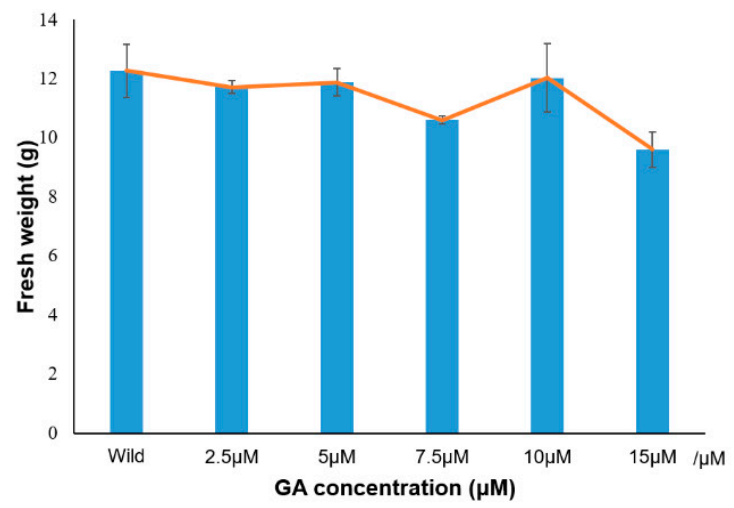

\section{(b)}

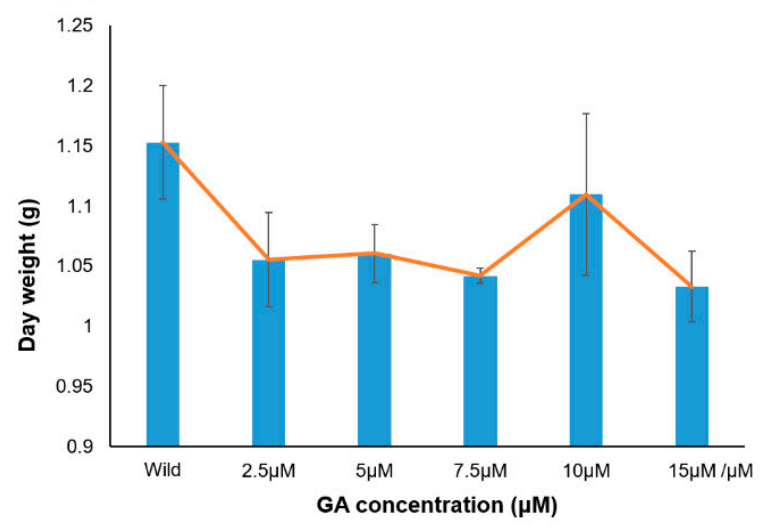

Figure 7. Fresh and dry weight of ginseng hairy roots were treated with different concentrations. (a) Fresh weight of ginseng hairy roots after treatment for 33 days. (b) Dry weight of ginseng hairy roots after treatment for 33 days. Error bars show the standard error between three replicates performed.

(a)

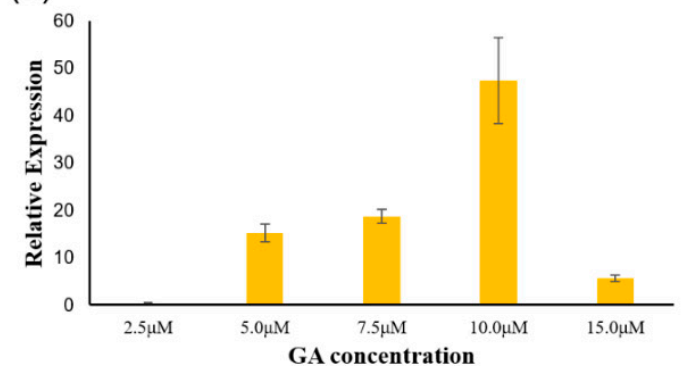

(c)

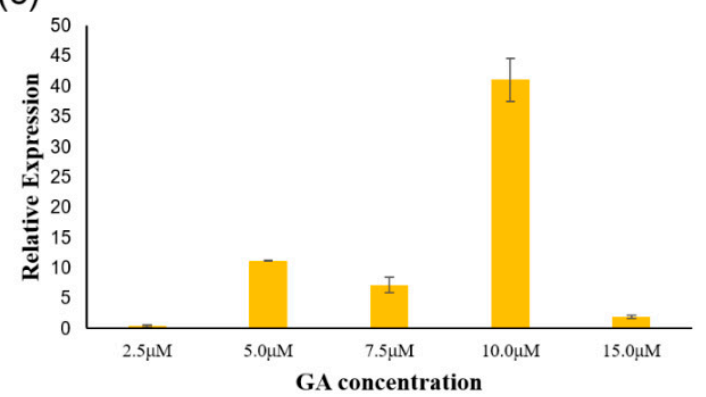

(b)

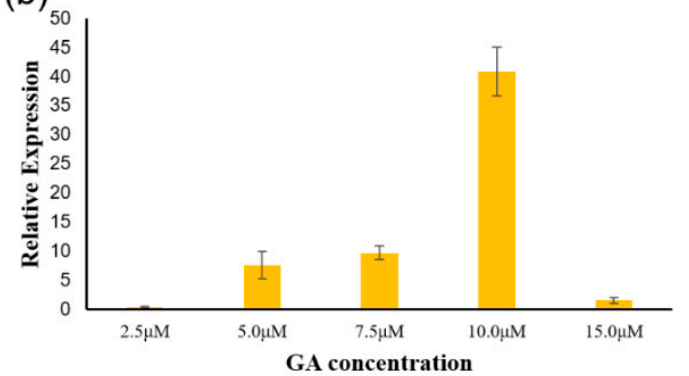

(d)

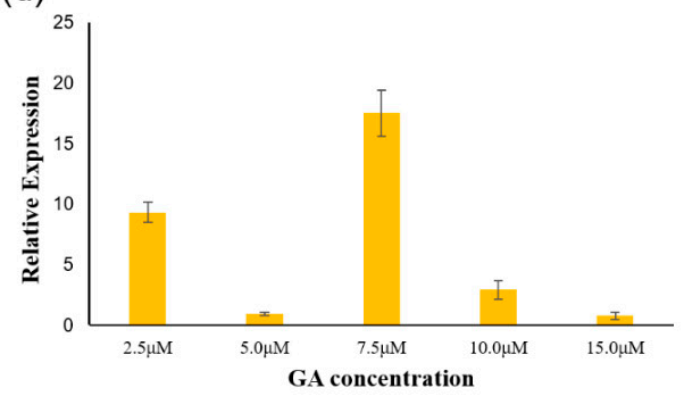

Figure 8. Relative expression levels of $P g G R A S$ genes in different concentration GA treatments by qPCR. (a) Expression of PgGRAS44-04 after GA treatment at different concentrations. (b) Expression of PgGRAS48-01 after GA treatment at different concentrations. (c) Expression of PgGRAS50-01 after GA treatment at different concentrations. (d) Expression of PgGRAS68-01 after GA treatment at different concentrations. The expression level of the control sample was normalized to one. Error bars show the standard error between three replicates performed. 


\section{Discussion}

\subsection{Function and Analysis of GRAS Transcription Factor Family}

Since transcription factors play a very important role in plants, a large number of TFs are analyzed in many species [59]. However, the analyses of the GRAS gene family have been performed far less than those of other transcription factor families, and the study of the GRAS gene family in ginseng has not been reported until now. In this study, 131 transcripts of 79 genes from the Jilin ginseng transcriptome data of Panax ginseng were analyzed. The number of genes isolated from plants such as Solanum lycopersicum (53) and Oryza sativa (60) was similar, but higher than that of GRAS in Arabidopsis thaliana (34). The identification of these genes can further fill the gap of ginseng resources in GenBank. According to the gene tree, it can be observed that the PgGRAS gene family has been well clustered with the GRAS genes of Arabidopsis thaliana, Oryza sativa and Solanum lycopersicum. These results suggest that the origins of the PgGRAS gene family can be traced back to the division between monocotyledons (Oryza sativa) and dicotyledons (Arabidopsis thaliana, Solanum lycopersicum and Panax ginseng).

Through the functional analysis of $P g G R A S$, we found that the functions of the $P g G R A S$ gene family have been largely differentiated. On the same level (Level 2), the PgGRAS gene family was categorized into at least eight GO functional subcategories and distributed across all three major GO functions, namely Biological Processes (BP), Molecular Function (MF) and Cellular Components (CC). There were great differences among the functions of the members of the PgGRAS gene family. However, according to the analysis, it was found that there were similar expression patterns among family members. Therefore, we can confirm that there is a network of co-expression among PgGRAS genes. Notwithstanding this, the function and expression activity of the PgGRAS genes have been greatly diversified. Most genes tend to have related expressions, and they also form a co-expression network, even if the correlation is limited. This indicates that members of the gene family maintain functional coordination or interaction to a certain extent. The formation of numerous mutual networks within the gene family provides a series of evidence for this inference, and it further confirmed the functional differentiation among members of the PgGRAS gene family.

\subsection{Response of DELLA Sub-Family to Gibberellin and Its Effect on Ginsenosides}

Expression analysis by qPCR revealed that four $\operatorname{Pg} G R A S$ genes were involved in the GA regulatory pathway. According to previous reports, many families of transcription factors are involved in the regulation of hormones, and GA is involved in many aspects of plant growth and development, such as seed growth and development, stem elongation and flower development. After the comprehensive analysis of all the qPCR data (Figure 8), we found that four genes belonging to the DELLA sub-family (PgGRAS44-04, PgGRAS48-01, PgGRAS50-01 and PgGRAS68-01) had significant changes in response to GA expression levels, suggesting that these genes could be involved in GA signaling and stress response.

GA as a plant hormone (gibberellin) is mainly synthesized in fruits or seeds, elongated stem ends and root organs of higher plants. Additionally, it promotes the germination of plant seeds, breaks dormancy and promotes stem and root growth. In addition, studies have found that GA and jasmonic acid (JA) interact with each other, which can play a synergistic role in plant development and an antagonistic role in plant defense. Predictions from other species indicate that GRAS proteins are an important component of the GA signaling pathway. In Arabidopsis, the DELLA protein acts as a repressor for GA response to plant growth $[60,61]$. Since studies have shown that the exogenous addition of JA can promote the content of ginsenosides in ginseng, it is speculated that there is a relationship between the synthesis of GA and ginsenoside content. The analysis of bioinformatics provides a basic information for studying the molecular regulation of GRAS proteins during ginseng development. This study provides ideas for future studies for the interaction between GA synthesis and the signal pathway and GA and ginsenoside synthesis in ginseng, and further improves ginseng resources. 


\section{Conclusions}

In this study, 59 GRAS genes ( $P g G R A S$ ) are found in Jilin ginseng and divided into 13 sub-families. These $59 \mathrm{PgGRAS}$ genes have a correlation of expression and form a co-expression network to function. We identified that four PgGRAS genes belonging to the DELLA sub-family (PgGRAS44-04, PgGRAS48-01, PgGRAS50-01 and PgGRAS68-01) had significant changes in expression levels in response to GA. The result of qPCR suggests that these genes can be involved in GA signaling and stress response. In summary, our results provide valuable data for further exploring the candidate PgGRAS genes of GA signaling in ginseng, especially their roles in ginseng hairy root development and stress response.

Supplementary Materials: The following are available online at http://www.mdpi.com/2223-7747/9/2/190/s1, Table S1. The transcript sequences of the PgGRAS genes identified in this study. Table S2. The transcript protein sequences of the $P g G R A S$ genes in this study. Table S3. The identified genes used as evolutionary tree for $P g G R A S$ genes evolutionary analysis. Table S4. The expressions of PgGRAS transcripts (TPM) in 14 tissues, 42 farmers' cultivars and four different year-old roots. Table S5. The classification, annotation and GO functional categorization of the PgGRAS gene transcripts.

Author Contributions: Conceptualization, N.W. and K.W.; methodology, N.W.; software, N.W., L.L. and L.Z.; validation, N.W., L.L. and Y.J. (Yang Jiang); formal analysis, N.W. and K.W.; investigation, Y.J. (Yue Jiang), S.L. and N.W.; resources, Y.W. (Yi Wang) and M.Z. (Meiping Zhang); data curation, Y.W. (Yanfang Wang), Y.W. (Yi Wang) and M.Z. (Meiping Zhang); writing — original draft preparation, N.W. and K.W.; writing-review and editing, N.W. and K.W.; visualization, M.Z. (Mingzhu Zhao); supervision, K.W., Y.W. (Yi Wang) and M.Z. (Meiping Zhang); project administration, K.W., Y.W. (Yi Wang) and M.Z. (Meiping Zhang); funding acquisition, Y.W. (Yanfang Wang), Y.S., Y.W. (Yi Wang) and M.Z. (Meiping Zhang) All authors have read and agreed to the published version of the manuscript.

Funding: This work was supported by an award from the China 863 Project (2013AA102604-3), the Bureau of Science and Technology of Jilin Province (20170101010JC, 20180414077GH, 20180101027JC, 20190201171JC and 20190201264JC) and the Development and Reform Commission of Jilin Province (2016C064 and 2018C047-3).

Conflicts of Interest: The authors declare no conflict of interest.

Additional Information: The sequences of the PgGRAS genes have been submitted at NCBI under BioProject RJNA302556.

\section{References}

1. Wang, J.; Gao, W.Y.; Zhang, J.; Zuo, B.M.; Zhang, L.M.; Huang, L.Q. Advances in study of ginsenoside biosynthesis pathway in Panax ginseng C. A. Meyer. Acta Physiol. Plant. 2012, 34, 397-403. [CrossRef]

2. Zhang, B.; Liu, J.; Yang, Z.E.; Chen, E.Y.; Zhang, C.J.; Zhang, X.Y.; Li, F.G. Genome-wide analysis of GRAS transcription factor gene family in Gossypium hirsutum L. BMC Genom. 2018, 19, 348. [CrossRef] [PubMed]

3. Ho, P.T.; Molinero, R.N.; Fariña, F.D.; Villena, D.M.; Garcíae, G.J. Identification and expression analysis of GRAS transcription factor genes involved in the control of Arbuscular mycorrhizal development in tomato. Front. Plant Sci. 2019, 10, 268.

4. Liu, B.L.; Sun, Y.; Xue, J.A.; Li, R.Z. Genome-wide characterization and expression analysis of GRAS gene family in pepper (Capsicum annuum L.). PeerJ 2018, 6, e4796. [CrossRef]

5. Dubos, C.; Stracke, R.; Grotewold, E.; Weisshaar, B.; Martin, C.; Lepiniec, L. MYB transcription factors in Arabidopsis. Trends Plant Sci. 2010, 15, 573-581. [CrossRef]

6. Jakoby, M.; Weisshaar, B.; Dröge-Laser, W.; Vicente-Carbajosa, J.; Tiedemann, J.; Kroj, T.; Parcy, F. bZIP transcription factors in Arabidopsis. Trends Plant Sci. 2002, 7, 106-111. [CrossRef]

7. Jiang, J.J.; Ma, S.H.; Ye, N.H.; Jiang, M.; Cao, J.S.; Zhang, J.H. WRKY transcription factors in plant responses to stresses. J. Integr. Plant Biol. 2017, 59, 86-101. [CrossRef]

8. Li, M.Y.; Liu, J.X.; Hao, J.N.; Feng, K.; Duan, A.Q.; Yang, Q.Q.; Xu, Z.S.; Xiong, A.S. Genomic identification of $\mathrm{AP} 2 / \mathrm{ERF}$ transcription factors and functional characterization of two cold resistance-related AP2/ERF genes in celery (Apium graveolens L.). Planta 2019, 250, 1265-1280. [CrossRef]

9. Cenci, A.; Rouard, M. Evolutionary analyses of GRAS transcription factors in angiosperms. Front. Plant Sci. 2017, 8, 273. [CrossRef]

10. Silverstone, A.L.; Ciampaglio, C.N.; Sun, T.P. The Arabidopsis RGA gene encodes a transcriptional regulator repressing the gibberellin signal transduction pathway. Plant Cell 1998, 10, 155-169. [CrossRef] 
11. Pysh, L.D.; Wysocka-Diller, J.W.; Camilleri, C.; Bouchez, D.; Benfey, P.N. The GRAS gene family in Arabidopsis: Sequence characterization and basic expression analysis of the SCARECROW-LIKE genes. Plant J. 1999, 18, 111-119. [CrossRef] [PubMed]

12. Zhang, H.L.; Cao, Y.P.; Shang, C.; Li, J.K.; Wang, J.L.; Wu, Z.Y.; Ma, L.C.; Qi, T.X.; Fu, C.X.; Bai, Z.T.; et al. Genome-wide characterization of GRAS family genes in Medicago truncatula reveals their evolutionary dynamics and functional diversification. PLoS ONE 2017, 12, e0185439. [CrossRef] [PubMed]

13. Bolle, C. The role of GRAS proteins in plant signal transduction and development. Planta 2004, 218, 683-692. [CrossRef] [PubMed]

14. Lee, M.H.; Kim, B.; Song, S.K.; Heo, J.O.; Yu, N.; Lee, S.A.; Kim, M.; Kim, D.G.; Sohn, S.O.; Lim, C.E.; et al. Large-scale analysis of the GRAS gene family in Arabidopsis thaliana. Plant Mol. Biol. 2018, 67, 659-670. [CrossRef] [PubMed]

15. Sun, X.L.; Xue, B.; Jones, W.T.; Rikkerink, E.; Dunker, A.K.; Uversky, V.N. A functionally required unfoldome from the plant kingdom: Intrinsically disordered $\mathrm{N}$-terminal domains of GRAS proteins are involved in molecular recognition during plant development. Plant Mol. Biol. 2011, 77, 205-223. [CrossRef]

16. Liu, X.Y.; Widmer, A. Genome-wide comparative analysis of the GRAS gene family in Populus, Arabidopsis and rice. Plant Mol. Biol. Report. 2014, 32, 1129-1145. [CrossRef]

17. Bolle, C.; Koncz, C.; Chua, N.H. PAT1, a new member of the GRAS family, is involved in phytochrome a signal transduction. Genes Dev. 2000, 14, 1269-1278.

18. Engstrom, E.M. Phylogenetic analysis of GRAS proteins from moss, lycophyte and vascular plant lineages reveals that GRAS genes arose and underwent substantial diversifification in the ancestral lineage common to bryophytes and vascular plants. Plant Signal. Behav. 2011, 6, 850-854. [CrossRef]

19. Wang, Y.; Shi, S.L.; Zhou, Y.; Yang, J.; Tang, X.Q. Genome-wide identification and characterization of GRAS transcription factors in sacred lotus (Nelumbo nucifera). PeerJ 2016, 4, e2388. [CrossRef]

20. Miyashima, S.; Hashimoto, T.; Nakajima, K. ARGONAUTE1 acts in Arabidopsis root radial pattern formation independently of the SHR/SCR pathway. Plant Cell Physiol. 2009, 50, 626-634. [CrossRef]

21. Smit, P.; Raedts, J.; Portyanko, V.; Debellé, F.; Gough, C.; Bisseling, T.; Geurts, R. NSP1 of the GRAS protein family is essential for rhizobial Nod factor-induced transcription. Science 2005, 308, 1789-1791. [CrossRef] [PubMed]

22. Ma, H.S.; Liang, D.; Shuai, P.; Xia, X.L.; Yin, W.L. The salt-and drought-inducible poplar GRAS protein SCL7 confers salt and drought tolerance in Arabidopsis thaliana. J. Exp. Bot. 2010, 61, 4011-4019. [CrossRef] [PubMed]

23. Tong, H.N.; Jin, Y.; Liu, W.B.; Li, F.; Fang, J.; Yin, Y.H.; Qian, Q.; Zhu, L.H.; Chu, C.C. DWARF ANDLOW-TILLERING, a new member of the GRAS family, plays positive roles in brassinosteroid signaling in rice. Plant J. 2009, 58, 803-816. [CrossRef] [PubMed]

24. Xue, L.; Cui, H.T.; Buer, B.; Vijayakumar, V.; Delaux, P.M.; Junkermann, S.; Bucher, M. Network of GRAS transcription factors involved in the control of arbuscule development in Lotus japonicus. Plant Physiol. 2015, 167, 854-871. [CrossRef] [PubMed]

25. Heck, C.; Kuhn, H.; Heidt, S.; Walter, S.; Rieger, N.; Requena, N. Symbiotic fungi control plant root cortex development through the novel GRAS transcription factor MIG1. Curr. Biol. 2016, 26, 2770-2778. [CrossRef] [PubMed]

26. Dill, A.; Jung, H.S.; Sun, T.P. The DELLA motif is essential for gibberellin-induced degradation of RGA. Proc. Natl. Acad. Sci. USA 2001, 98, 14162-14167. [CrossRef]

27. Zhang, D.P.; Iyer, L.M.; Aravind, L. Bacterial GRAS domain proteins throw new light on gibberellic acid response mechanisms. Bioinformatics 2012, 28, 2407-2411. [CrossRef]

28. Finn, R.D.; Bateman, A.; Clements, J.; Coggill, P.; Eberhardt, R.Y.; Eddy, S.R.; Heger, A.; Hetherington, K.; Holm, L.; Mistry, J.; et al. Pfam: The protein families database. Nucleic Acids Res. 2014, 42, D222-D230. [CrossRef]

29. Finn, R.D.; Clements, J.; Eddy, S.R. HMMER Web Server: Interactive Sequence Similarity Searching. Nucleic Acids Res. 2011, 39, W29-W37. [CrossRef]

30. Grabherr, M.G.; Haas, B.J.; Yassour, M.; Levin, J.Z.; Thompson, D.A.; Amit, I.; Adiconis, X.; Fan, L.; Raychowdhury, R.; Zeng, Q.; et al. Full-length transcriptome assembly from RNA-Seq data without a reference genome. Nat. Biotechnol. 2011, 29, 644. [CrossRef] 
31. Haas, B.J.; Papanicolaou, A.; Yassour, M.; Grabherr, M.; Blood, P.D.; Bowden, J.; Couger, M.B.; Eccles, D.; Li, B.; Lieber, M.; et al. De novo transcript sequence reconstruction from RNA-seq using the Trinity platform for reference generation and analysis. Nat. Protoc. 2013, 8, 1494. [CrossRef] [PubMed]

32. Gasteiger, E.; Hoogland, C.; Gattiker, A.; Duvaud, S.; Wilkins, M.R.; Appel, R.D.; Bairoch, A. Protein identification and analysis tools on the ExPASy server. In The Proteomics Protocols Handbook; Human Press: Totowa, NJ, USA, 2005; pp. 571-607.

33. Bailey, T.L.; Williams, N.; Misleh, C.; Li, W.W. MEME: Discovering and analyzing DNA and protein sequence motifs. Nucleic Acids Res. 2006, 34, W369-W373. [CrossRef] [PubMed]

34. Kumar, S.; Stecher, G.; Tamura, K. MEGA 7: Molecular evolutionary genetics analysis version 7.0 for bigger datasets. Mol. Biol. Evol. 2016, 33, 1870-1874. [CrossRef] [PubMed]

35. Subramanian, B.; Gao, S.H.; Lercher, M.J.; Hu, S.N.; Chen, W.H. Evolview v3: A webserver for visualization, annotation, and management of phylogenetic trees. Nucleic Acids Res. 2019, 47, W270-W275. [CrossRef] [PubMed]

36. Chen, C.J.; Xia, R.; Chen, H.; He, Y.H. TBtools, a Toolkit for Biologists integrating various HTS-data handling tools with a user-friendly interface. BioRxiv 2018, 289660.

37. Ashburner, M.; Ball, C.A.; Blake, J.A.; Botstein, D.; Butler, H.; Cherry, J.M.; Davis, A.P.; Dolinski, K.; Dwight, S.S.; Eppig, J.T.; et al. Gene ontology: Tool for the unification of biology. Nat. Genet. 2000, 25, 25. [CrossRef]

38. Theocharidis, A.; Van, D.S.; Enright, A.J.; Freeman, T.C. Network visualization and analysis of gene expression data using BioLayout Express (3D). Nat. Protoc. 2009, 4, 1535. [CrossRef]

39. Jong, M.D.; Mariani, C.; Vriezen, W.H. The role of auxin and gibberellin in tomato fruit set. J. Exp. Bot. 2009, 60, 1523-1532. [CrossRef]

40. Ma, Z.; Hu, X.; Cai, W.; Huang, W.; Zhou, X.; Luo, Q.; Yang, H.; Wang, J.; Huang, J. Arabidopsis miR171-targeted scarecrow-like proteins bind to GT cis-elements and mediate gibberellin-regulated chlorophyll biosynthesis under light conditions. PLoS Genet. 2014, 10, e1004519. [CrossRef]

41. Li, L.; Wang, K.Y.; Zhao, M.Z.; Li, S.K.; Jiang, Y.; Zhu, L.; Chen, J.; Wang, Y.F.; Sun, C.Y.; Chen, P.; et al. Selection and validation of reference genes desirable for gene expression analysis by qRT-PCR in MeJA-treated ginseng hairy roots. PLoS ONE 2019, 14, e0226168. [CrossRef]

42. Pfaffl, M.W. A new mathematical model for relative quantification in real-time RT-PCR. Nucleic Acids Res. 2001, 29, e45. [CrossRef] [PubMed]

43. Gao, L.; Gonda, I.; Sun, H.H.; Ma, Q.Y.; Bao, K.; Tieman, D.M.; Burzynski-Chang, E.A.; Fish, T.L.; Stromberg, K.A.; Sacks, G.L.; et al. The tomato pan-genome uncovers new genes and a rare allele regulating fruit flavor. Nat. Genet. 2019, 51, 1044. [CrossRef] [PubMed]

44. Zhang, M.P.; Wu, Y.H.; Lee, M.K.; Liu, Y.H.; Rong, Y.; Santos, T.S.; Wu, C.C.; Xie, F.M.; Nelson, R.L.; Zhang, H.B. Numbers of genes in the NBS and RLK families vary by more than four-fold within a plant species and are regulated by multiple factors. Nucleic Acids Res. 2010, 38, 6513-6525. [CrossRef] [PubMed]

45. Xu, W.; Chen, Z.X.; Ahmed, N.; Han, B.; Cui, Q.H.; Liu, A.Z. Genome-wide identification, evolutionary analysis, and stress responses of the GRAS gene family in castor beans. Int. J. Mol. Sci. 2016, 17, 1004. [CrossRef] [PubMed]

46. Grimplet, J.; Agudelo-Romero, P.; Teixeira, R.T.; Martinez-Zapater, J.M.; Fortes, A.M. Structural and functional analysis of the GRAS gene family in grapevine indicates a role of GRAS proteins in the control of development and stress responses. Front. Plant Sci. 2016, 7, 353. [CrossRef]

47. Gu, X. Maximum-likelihood approach for gene family evolution under functional divergence. Mol. Biol. Evol. 2001, 18, 453-464. [CrossRef]

48. Fan, S.; Zhang, D.; Gao, C.; Zhao, M.; Wu, H.; Li, Y.; Shen, Y.; Han, M. Identification, classification, and expression analysis of GRAS gene family in Malus domestica. Front. Physiol. 2017, 8, 253. [CrossRef]

49. Morohashi, K.; Minami, M.; Takase, H.; Hotta, Y.; Hiratsuka, K. Isolation and characterization of a novel GRAS gene that regulates meiosis-associated gene expression. J. Biol. Chem. 2003, 278, 20865-20873. [CrossRef]

50. Torres-Galea, P.; Hirtreiter, B.; Bolle, C. Two GRAS proteins, SCARECROW-LIKE21 and PHYTOCHROME A SIGNAL TRANSDUCTION1, function cooperatively in phytochrome a signal transduction. Plant Physiol. 2013, 161, 291-304. [CrossRef] 
51. Li, W.; Wu, J.; Weng, S.; Zhang, Y.; Zhang, D.; Shi, C. Identification and characterization of dwarf 62, a loss-of-function mutation in DLT/OsGRAS-32 affecting gibberellin metabolism in rice. Planta 2010, 232, 1383-1396. [CrossRef]

52. Zhang, Y.; Liu, Z.; Liu, J.; Lin, S.; Wang, J.; Lin, W.; Xu, W. GA-DELLA pathway is involved in regulation of nitrogen deficiency-induced anthocyanin accumulation. Plant Cell Rep. 2017, 36, 557-569. [CrossRef] [PubMed]

53. Guo, Y.Y.; Wu, H.Y.; Li, X.; Li, Q.; Zhao, X.Y.; Duan, X.Q.; An, Y.R.; Lv, W.; An, H.L. Identification and expression of GRAS family genes in maize (Zea mays L.). PLoS ONE 2017, 12, e0185418. [CrossRef] [PubMed]

54. Yin, R.; Zhao, M.Z.; Wang, K.Y.; Lin, Y.P.; Wang, Y.F.; Sun, C.Y.; Wang, Y.; Zhang, M.P. Functional differentiation and spatial-temporal co-expression networks of the NBS-encoding gene family in Jilin ginseng, Panax ginseng C. A. Meyer. PLoS ONE 2017, 12, e0181596. [CrossRef] [PubMed]

55. Wang, K.Y.; Jiang, S.C.; Sun, C.Y.; Lin, Y.P.; Yin, R.; Wang, Y.; Zhang, M.P. The spatial and temporal transcriptomic landscapes of ginseng, Panax ginseng C. A. Meyer. Sci. Rep. 2015, 5, 18283. [CrossRef]

56. Lin, Y.P.; Wang, K.Y.; Li, X.Y.; Sun, C.Y.; Yin, R.; Wang, Y.F.; Wang, Y.; Zhang, M.P. Evolution, functional differentiation, and co-expression of the RLK gene family revealed in Jilin ginseng, Panax ginseng C. A. Meyer. Mol. Genet. Genom. 2018, 293, 845-859. [CrossRef]

57. Itoh, H.; Ueguchi-Tanaka, M.; Sato, Y.; Ashikari, M.; Matsuoka, M. The gibberellin signaling pathway is regulated by the appearance and disappearance of SLENDER RICE1 in nuclei. Plant Cell 2002, 14, 57-70. [CrossRef]

58. Sun, T.P.; Gubler, F. Molecular mechanism of gibberellin signaling in plants. Annu. Rev. Plant Biol. 2004, 55, 197-223. [CrossRef]

59. Zeng, X.; Ling, H.; Chen, X.M.; Guo, S.X. Genome-wide identification, phylogeny and function analysis of GRAS gene family in Dendrobium catenatum (Orchidaceae). Gene 2019, 705, 5-15. [CrossRef] [PubMed]

60. Park, J.; Nguyen, K.T.; Park, E.; Jeon, J.S.; Choi, G. DELLA proteins and their interacting RING Finger proteins repress gibberellin responses by binding to the promoters of a subset of gibberellin-responsive genes in Arabidopsis. Plant Cell 2013, 25, 927-943. [CrossRef]

61. Tyler, L.; Thomas, S.G.; Hu, J.H.; Dill, A.; Alonso, J.M.; Ecker, J.R.; Sun, T.P. DELLA proteins and gibberellin-regulated seed germination and floral development in Arabidopsis. Plant Physiol. 2004, 135, 1008-1019. [CrossRef] 